\title{
ESTUDIOS BREVES
}

\section{CONSTRUCCIÓN DEL CONOCIMIENTO EN EDUCACIÓN SUPERIOR A TRAVÉS DEL APRENDIZAJE POR PROYECTOS}

\section{KNOWLEDGE BUILDING IN HIGHER EDUCATION THROUGH PROJECT- BASED LEARNING}

Juan Jesús Torres Gordillo*

Universidad de Sevilla

\begin{abstract}
RESUMEN
Este estudio presenta los resultados de la implantación del método del aprendizaje basado en proyectos (ABP) y del proceso de construcción del conocimiento en un contexto auténtico con alumnos de $4^{\circ}$ curso de Pedagogía en la Universidad de Sevilla.
\end{abstract}

Palabras clave: Aprendizaje basado en proyectos, aprendizaje universitario, actividades auténticas, construcción de conocimiento, ambientes de aprendizaje online

\begin{abstract}
The purpose is to share our experience using a project-based learning method within an authentic context with fourth-level undergraduate students at the Faculty of Science of Education at the University of Seville (Spain). We intended to study how the implantation of this method and knowledge building processes are delivered. We focused on knowing the results of this process in the students' own opinions. Research techniques were content analysis for open-ended survey questions, and SPSS contingency tables for closed-ended questions. Conclusions contribute to our knowledge of the advantages and problems of implanting this methodology to support the knowledge building processes.
\end{abstract}

Key words: Project-based learning, University learning, authentic tasks, knowledge building, online learning environments.

\section{Introducción}

El artículo recoge el estudio del proceso de construcción del conocimiento desde la puesta en práctica del aprendizaje por proyectos colaborativos (ABP) con estudiantes de Pedagogía de la Universidad de Sevilla. Se utilizó la plataforma Blackboard para animar el trabajo online como complemento a lo presencial (blended-learning), dentro de ambientes de aprendizaje auténticos (Reeves, Herrington y Oliver, 2002; Herrington, Oliver y Reeves, 2003; Herrington et al., 2004). 
* Dtor. En Pedagogía por la Universidad de Sevilla. Es miembro del Grupo IDEA (HUM-423, http://prometeo.us.es/idea). Su interés actual se centra en los procesos metodológicos y evaluativos innovadores de enseñanza-aprendizaje y de investigación en Educación Superior en e-Learning. juani@us.es

Una de las ideas principales por las que surge el ABP es para superar la metodología tradicional basada en la memorización, que vuelve irrelevante el trabajo en la realidad exterior (ITESM, s.f.).

El ABP es una metodología didáctica centrada en los estudiantes, donde éstos trabajan en grupos y aprenden investigando sobre proyectos basados en su futuro trabajo como profesionales (Reverte et al., 2007). Fomenta habilidades como la autonomía, la autoevaluación, la planificación del tiempo, el trabajo por proyectos, la expresión oral y escrita, así como la motivación y el rendimiento.

Como recuerdan Lucas et al. (2006), el objetivo del método no está tanto en la resolución del problema, como en utilizar éste como base para identificar los temas a estudiar; la mayor parte del aprendizaje ocurre en el contexto de pequeños grupos de trabajo; el conflicto cognitivo al enfrentar cada nueva situación estimula el aprendizaje (ITESM, s.f.); y el profesor actúa como facilitador, y estimula el razonamiento a través de la discusión y el análisis crítico (Lucas et al., 2006).

Algunas razones por las que usar el ABP son (Taplin, 2000): un aprendizaje autodirigido para mantenerse al día en el conocimiento; el aprendizaje es más efectivo cuando se estimula a adquirir nuevos conocimientos por sí mismo, a través del razonamiento y procesamiento de la información; se desarrollan destrezas de pensamiento de nivel superior; puede mejorar la capacidad del estudiante para aplicar las destrezas y conocimientos que ha generado a situaciones desconocidas o poco familiares. Hoffman y Ritchie (1997) añaden que se tiende a estudiar por significados más que a reproducir materiales del docente. Incrementa la autodirección e integración del conocimiento, y elimina la transferencia pasiva de información propia del aprendizaje tradicional (ITESM, s.f.).

\section{Método}

El objetivo es conocer los resultados de la implantación del ABP desde la propia opinión de los estudiantes. El estudio fue realizado con dos aulas de $4^{\circ}$ curso durante $2007-08$ con 68 estudiantes.

Diseñamos una encuesta de valoración ad hoc, anónima y voluntaria, con 9 preguntas de opción múltiple y 6 abiertas. Las cerradas se analizaron a través de tablas de contingencia con SPSS. Para las abiertas se empleó el análisis de contenido. La tarea principal en la asignatura consistía en diseñar, implementar y evaluar un proyecto de intervención psicopedagógica sobre un tema elegido por cada grupo. Se ofreció un constante apoyo tutorial (enseñanza tutorada), tanto en el aula presencial, como de forma asíncrona a través de los foros generales y específicos.

\section{Resultados}


Usada para conocer la opinión de los alumnos, presentamos los resultados de las preguntas cerradas a través del análisis de tablas de contingencia. La escala de respuestas era: Mucho, Bastante, Normal y Poco. Empleamos frecuencias absolutas y relativas. Establecimos tres tipos de cruces.

En el primer cruce nos interesaba saber si una diferente formación previa del alumnado podría llevarnos a obtener distintos niveles de opinión. Pero no ha sido una variable diferenciadora, ya que la mayoría (85\%) consideró que sólo tenía unos conocimientos suficientes para enfrentarse a la asignatura. Dos de cada tres alumnos del total han valorado muy positivamente la tutorización del profesor y que la plataforma les ha ayudado a mejorar el seguimiento de la asignatura.

Las clases y el trabajo en grupo han ayudado bastante a conseguir los objetivos al 55\% con conocimientos previos suficientes. Tan sólo el 15\% consideró que las clases ayudaron poco. El dato más significativo lo apreciamos respecto a que la metodología tan sólo fue bastante adecuada para un $39 \%$, normal para el $31 \%$ y poco para un $15 \%$ de los que presentan nociones suficientes de partida. Los de bastantes conocimientos tienden a la normalidad en la concepción del APB.

La mitad ha adquirido los contenidos de forma normal, y sólo un tercio dice dominarlos mucho o bastante. No obstante, el $70 \%$ con conocimientos normales asume haber conseguido los objetivos de forma muy satisfactoria, y satisfactoria para los que partían con mayor bagaje competencial. Y la asignatura resultó bastante exigente, independientemente del nivel previo de conocimientos.

La segunda cuestión comprobó si la percepción del nivel de exigencia en la asignatura establecía diferencias en otros ítems. La alta exigencia también se asocia a una alta valoración del trabajo de tutorización docente o del trabajo en grupo para más del $70 \%$, y normales para el resto. Las clases y la plataforma ayudaron bastante a más de la mitad de los que vieron exigente la materia.

Los contenidos y la metodología presentan los porcentajes más moderados. La alta exigencia se relaciona con una alta satisfacción para con la metodología en el 39\%, mientras el mismo porcentaje se repite para los que la apreciaron normal. Sobre los contenidos, la fuerza dominante está en la respuesta normal, con la mitad de los estudiantes, llegando al 30\% los que admiten dominarlos bien. A pesar de esto, como ocurría en el primer cruce, hay casi unanimidad (85\%) para afirmar que se han obtenido los objetivos de manera bastante adecuada entre los que lo han considerado exigente.

En el último cruce indagamos si con distintos niveles de valoración del rol del profesor, variaban las respuestas para el resto de variables. Esta hipótesis tiene unos resultados muy claros, dado que la valoración alta o muy alta del profesor alcanza el $82 \%$. Uno de cada dos estudiantes tiene una opinión muy favorable hacia el trabajo docente realizado y admite que las clases y el trabajo en grupo les han servido bastante o mucho. El $20 \%$ y el $27 \%$ con buena valoración del docente entienden que las clases y el trabajo grupal, respectivamente, fueron normales. Y sólo a otro $20 \%$ les gustaron las clases valorando con normalidad al docente. 
Un tercio del total presenta valores altos para el profesor y la metodología, y el mismo porcentaje respecto a los contenidos. El 27\% valora bastante el seguimiento docente siendo la metodología y la adquisición de contenidos normales. Así, la valoración de la metodología y el dominio de los contenidos tienen los cruces porcentuales más moderados. Por último, el $73 \%$ con una percepción buena del trabajo docente piensa que ha alcanzado bastante bien los objetivos. El resto cree que posee bastante las competencias previstas, pero sólo percibe el rol del profesor como normal.

Encuesta de evaluación final: preguntas abiertas

El análisis de contenido se realizó sobre las respuestas a las seis preguntas abiertas. A un solo alumno no gustó la metodología $A B P$. Afirman que es exigente y complicada a veces, y requiere implicación, pero destacan la posibilidad de conocer otros puntos de vista, ser buena para los objetivos marcados, muy interesante e útil, y especialmente bastante efectiva al acercarlos a la realidad práctica. Otros calificativos son: muy positiva, práctica, coherente, lógica, enriquecedora y motivadora, que permite autonomía y trabajar muy bien los contenidos para conseguir un mejor aprendizaje y más duradero que otras de corte tradicional basadas en la lección magistral.

Con relación a los puntos fuertes del ABP, apuestan sobremanera por el trabajo grupal colaborativo y experimentar una práctica educativa real con aplicación directa de la teoría a la praxis. Resaltan la autonomía, la implicación en el propio aprendizaje significativo, la resolución de problemas y toma de decisiones, y la constancia y seguimiento. A todo ello se unen las ventajas de las TIC.

Aunque casi el $30 \%$ no señaló puntos débiles, se indicaron la falta de tiempo para tanto trabajo y las dificultades propias del trabajo grupal (desorganización, diversidad de objetivos entre alumnos...).

El $25 \%$ dijo no haber tenido dificultades en la aplicación del ABP, mientras las del resto son dispersas y poco relacionadas con el ABP (relativas al tiempo y mala organización del grupo).

Respecto a las propuestas de mejora tras la experiencia de un año, el $42 \%$ no describió nada o afirmó: "no lo sé". Las respuestas son variadas, aunque algunas son difíciles de asumir al ser características propias del ABP (como una dinámica más teórico-práctica, realizar las prácticas de otra forma, no dar tanta libertad...) o directamente inviables, como aumentar el $\mathrm{n}^{\circ}$ de créditos. Otras propusieron menos exigencia. En general se relacionan poco con el ABP, y sí con dificultades personales como I agobios, ansiedad... Finalmente, no hubo sugerencias significativas.

\section{Conclusiones}

Los resultados nos aproximan a nuestras expectativas iniciales, puesto que el alumnado ha entendido, no sin dificultades, la forma de trabajo por proyectos. Como obtenían Lucas et al. (2006), ITESM (s.f.) y Reverte et al. 
(2007), toman un rol más activo y responsable en la construcción de su aprendizaje. Les ha permitido mayor autonomía de acción y de toma de decisiones sobre su formación.

La tutorización del profesor adquiere un papel relevante para construir conocimiento y servir de guía en el proceso de trabajo en equipo. Ofrecer oportunidades de aprendizaje fuera del aula ha sido muy valorado por poder experimentar una práctica educativa real. Las tres fases por las que han pasado estos alumnos que se enfrentaban por primera vez al APB son: fase inicial de incredulidad, fase de dinámica con altibajos, y fase de velocidad de crucero. El rol dominante en algún miembro del grupo no ha sido lo más característico. Las dificultades para establecer una dinámica interna de trabajo han influido en ello. En muchos grupos predominó el aprendizaje estratégico, repartiendo las partes del trabajo con una puesta en común a posteriori.

Si bien en las preguntas cerradas los estudiantes han contestado que la metodología les había parecido más bien normal, y un menor porcentaje eran los que la consideraban muy satisfactoria, en las respuestas abiertas sí observamos casi por unanimidad mayor entusiasmo. Afirman que es muy exigente y necesita de mucho esfuerzo y horas de trabajo, pero es útil, interesante, coherente, lógica, motivadora, que permite autonomía y posibilidad de trabajo en grupo.

Las propuestas de mejora están relacionadas con problemas personales particulares. La principal es la mayor inversión de tiempo, por enfrentarse a nuevas situaciones de trabajo a las que estaban poco acostumbrados. Como establecía el ITESM (s.f.) o Taplin (2000), algunos han sufrido desconfianza al asumir roles y cierta ansiedad al sentir que no avanzaban como con otros métodos, creándoles dudas en la forma de trabajar. Este alto grado de libertad de acción ha descolocado inicialmente a muchos que esperaban asumir un rol más pasivo (Hoffman y Ritchie, 1997).

Una característica poco valorada es el incremento de tiempo y trabajo que el ABP les ha supuesto. Exponen esto por comparación a lo que vienen haciendo en otras asignaturas con métodos más tradicionales. Pero el ABP ha permitido superar estas prácticas desde la mayor satisfacción de los propios implicados, al igual que indican otros estudios (Reverte et al., 2007; Lucas et al., 2006; Cataldi y Cabero, 2006).

Aunque concluimos que la satisfacción con el ABP es alta, son necesarios más estudios similares, longitudinales y comparativos, para confirmar si se alcanza un mayor aprendizaje a largo plazo.

\section{Referencias bibliográficas}

Cataldi, Z. y Cabero, J. (2006). Los aportes de la Tecnología Informática al aprendizaje grupal interactivo: la resolución de problemas a través del foro de discusión y de chat. Píxel-Bit, 27.

Instituto Tecnológico y de Estudios Superiores de Monterrey (s.f.). El aprendizaje basado en problemas como técnica didáctica. Documento policopiado. México. 
Herrington, J., Oliver, R., \& Reeves, T.C. (2003). Patterns of engagement in authentic online learning environments. Australian Journal of Educational Technology, 19(1), 59-71.

Herrington, J., Reeves, T.C., Oliver, R., \& Woo, Y. (2004). Designing authentic activities in web-based courses. Journal of Computing in Higher Education, 16(1), 3-29.

Hoffman, B., \& Ritchie, D. (1997). Using multimedia to overcome the problems with problem based learning. Instructional Science, 25 (2), 97-115.

Lucas, M. et al. (2006). El aprendizaje basado en problemas aplicado a la asignatura de pregrado de Anestesiología, Reanimación y Terapéutica del Dolor. Revista Española de Anestesiología y Reanimación (SEDAR), 53 (7), 419-425.

Reeves, T.C., Herrington, J., \& Oliver, R. (2002). Authentic activities and online learning. In A. Goody, J. Herrington \& M. Northcote (Eds.). Proceedings of the 2002 Annual International Conference of the Higher Education Research and Development Society of Australasia (HERDSA), 562-567. Perth, Australia.

Reverte, J.R. et al. (2007). El aprendizaje basado en proyectos como modelo docente. Experiencia interdisciplinar y herramientas groupware. En: Actas de las XIII Jornadas de Enseñanza Universitaria de la Informática (JENUI'07). Madrid: Thomson Paraninfo.

Taplin, M. (2000). Problem-based learning in distance education: Practitioners' beliefs about an action learning project. Distance Education, 21(2), 278-299.

Fecha de recepción: 18-09-2009

Fecha de revisión: 09-01-2010

Fecha de aceptación: 19-01-2010 